\title{
DETERMINAÇÃO DA PRODUÇÃO DE COMPOSTOS VOLÁTEIS MICROALGAIS EM FUNÇÃO DA CURVA CINÉTICA DE CRESCIMENTO
}

\author{
PINHEIRO, P. N. ${ }^{1}$; VIEIRA, K. R. ${ }^{1}$; NEUENFELDT, N. H. ${ }^{1}$;ACOB-LOPES, E. ${ }^{1}$; ZEPKA, L. Q. ${ }^{1}$ \\ ${ }^{1}$ Universidade Federal de Santa Maria, Departamento de Ciência e Tecnologia em Alimentos \\ e-mail para contato: lqz@pq.cnpq.br
}

RESUMO - O objetivo do trabalho foi investigar o comportamento da fração volátil em diferentes fases de crescimento celular durante o cultivo heterotrófico de Phormidium autumnale. Os experimentos foram realizados em biorreator descontínuo com aeração contínua e ausência de luminosidade. Os compostos orgânicos voláteis foram isolados por microextração em fase sólida no headspace, analisados por cromatografia gasosa acoplada a um detector de massas (SPME-GC-MS). A amostragem foi realizada a cada 24 horas no período de 144 horas correspondendo às fases de crescimento microalgal. A análise dos resultados cinéticos apresentaram uma densidade máxima celular de $1050 \mathrm{mg} . \mathrm{L}^{-1}$ obtendo uma produtividade de biomassa de $13,19 \mathrm{mg} \cdot \mathrm{L}^{-1} \cdot \mathrm{h}^{-1}$, com uma velocidade específica máxima de crescimento celular 0,02h-1 ${ }^{1}$. Foram identificados 44 compostos ao longo das fases exponenciais, estacionária e de declínio, sendo que os majoritários foram Benzotiazol (28,78 $\left.\mu . L^{-1} ; 29,18 \mu . L^{-1} ; 44,38 \mu . L^{-1}\right)$, Ftalato de diisobutilo $\left(17,58 \mu . L^{-1} ; 12,88 \mu . L^{-1} ; 25,98 \mu . L^{-1}\right)$ e o 2-Nonanone $\left(1,1 \mu . \mathrm{L}^{-1} ; 4,77 \mu . \mathrm{L}^{-1} ; 7,74 \mu . \mathrm{L}^{-1}\right)$.

\section{INTRODUÇÃO}

As microalgas produzem uma vasta gama de metabólitos secundários de grande importância econômica, o cultivo dessas espécies está associado à diversidade da composição química, o potencial de crescimento em um biorreator com condições controladas e a sua capacidade de produzir estes metabólitos ativos em resposta ao estresse induzido por condições extremas de exposição (Plaza et al., 2009).

A Phormidium autumnale é apontada como uma espécie em potencial no tratamento de efluentes em sistemas que empregam microalgas, devido a sua tolerância a determinadas condições como temperaturas extremas e concentrações elevadas de nutrientes (SU et al., 2012), estas condições podem conferir estresse as microalgas levando-as produzir metabólitos secundários e excretá-las no meio (Shipin \& Meiring, 2005).

Compostos orgânicos voláteis (COV) são metabólitos secundários obtidos a partir de microalgas que poderiam ser usados como fonte alternativa de produtos farmacêuticos, aromas e fragrâncias a baixo custo (Havel \& Weuster-Botz, 2006), a utilização plena da fração volátil da biomassa microalgal pode representar uma melhor oferta de produtos para os diferentes tipos de indústrias (Santos et al., 2016).

Um volume considerável de informações a respeito dos metabólitos de microalgas tem sido gerado por décadas, porém pouco se relata sobre os eventos metabólicos complexos durante o crescimento e desenvolvimento das microalgas. Assim o objetivo do trabalho foi 
investigar o comportamento da fração volátil em diferentes fases de crescimento celular durante o experimento microalgal quando cultivada em água residuária.

\section{MATERIAL E MÉTODOS}

A microalga utilizada foi a Phormidium autumnale adaptada e mantida em água residuária diluída com meio sintético BG11 (Rippka et al., 1979), solução 1:1. A condição de manutenção utilizada foi de $25^{\circ} \mathrm{C}$ com intensidade luminosa constante de 1 klux. $\mathrm{O}$ experimento foi realizado em reator de coluna de bolhas. O sistema foi construído de vidro de borosilicato com diâmetro externo de $12,5 \mathrm{~cm}$ e altura de $16 \mathrm{~cm}$, com razão altura/diâmetro proporção igual a 1,28 e um volume nominal de 2,0L, a dispersão do reator consistiu em um difusor de ar com 2,5 centímetros de diâmetro localizado no interior do biorreator. $\mathrm{O}$ fluxo de ar controlado pelo medidor de fluxo (Kl-Key Instruments®,Ttrevose,PA, EUA) e a entrada de ar e a saída de gases foi filtrado com unidades filtrantes constituídas de membrana de polipropileno, com um diâmetro de poro de $0,22 \mu \mathrm{m}$ e o diâmetro total de $50 \mathrm{~mm}$ (Millex FG ${ }^{8}$, Billerica, MA, EUA). O biorreator juntamente com as unidades filtrantes, foi esterilizado em autoclave a $121^{\circ} \mathrm{C}$ durante 20 minutos. $\mathrm{O}$ experimento foi realizado em biorreator com regime de batelada, alimentado com $2,0 \mathrm{~L}$ de água residuária, oriundas do abate e processamento de aves e suínos. As condições experimentais foram as seguintes: concentração inicial do inóculo de $100 \mathrm{mg} . \mathrm{L}^{-1}$, a temperatura de $25^{\circ} \mathrm{C}$, o pH ajustado para 7,6, aeração de 1 VVM (volume de ar por volume de meio por minuto), ausência de luz e relação $\mathrm{C} / \mathrm{N}$ (carbono/nitrogênio) de 30. A amostragem foi realizada no tempo zero e a cada $24 \mathrm{~h}$ durante o crescimento celular totalizando um tempo de residência celular de $144 \mathrm{~h}$. A concentração celular foi determinada gravimetricamente por filtração de um volume

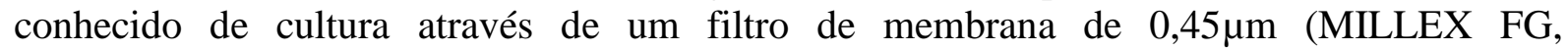
BILLRICA-MA, EUA) e secagem a $60^{\circ} \mathrm{C}$. As experiências foram realizadas em duplicada. Portanto, os dados referem-se ao valor médio das repetições. Os compostos voláteis formados no bioprocesso foram isolados pela técnica de micro extração em fase sólida aplicada em headspace (HS-SPME). A fibra de SPME de revestimento misto empregada foi a DVB/Car/PDMS $(50 / 30 \mu \mathrm{m} \times 20 \mathrm{~mm}$, Supelco Bellefonte, PA, USA), pré-condicionada conforme as recomendações fornecidas pelo fabricante. A temperatura de extração de $40^{\circ} \mathrm{C}$, com um tempo de equilíbrio de 5 minutos, após exposição da fibra por 45 minutos. A análise dos compostos voláteis foi realizada em um cromatógrafo a gás acoplado a um espectrômetro de massas (GC/MS Shimadzu QP-2010 Plus). As separações cromatográficas foram feitas em coluna capilar de sílica fundida DB-Wax, com $60 \mathrm{~m}$ de comprimento, 0,25 mm de diâmetro e $0,25 \mu \mathrm{m}$ de espessura de película (Chrompack Wax 52- CB). O gás de arraste utilizado foi o hélio com vazão constante de $1,6 \mathrm{ml} \cdot \mathrm{min}^{-1}$. A temperatura inicial da coluna de $35^{\circ} \mathrm{c}$, permanecendo por 5 minutos, após elevada até $220^{\circ} \mathrm{C}$ com gradiente de temperatura de $5^{\circ} \mathrm{C} / \mathrm{min}$, mantendo-se isotermicamente por 5 minutos. A interface GC/MS e da fonte de ionização foram mantidos a $250^{\circ} \mathrm{C}$. O detector de massas foi operado no modo de ionização por elétrons, com feixe de elétrons a $+70 \mathrm{eV}$. O analisador de massas do tipo quadrupolos foi utilizado no modo de varredura na faixa de 35 a $350 \mathrm{~m} / \mathrm{z}$. Os compostos foram identificados por comparação dos seus espectros de massa com os dados da biblioteca do GC-MS (NIST MS Search 2.0), paralelo à comparação dos índices de Retenção Linear calculados. 


\section{RESULTADO E DISCUSSÃO}

A Figura 1 mostra a curva de crescimento celular durante o cultivo heterotrófico, as fases exponencial (0h-96h), estacionária (96h-120h) e de declínio (120h-144h). Os dados cinéticos obtidos durante o experimento foram, densidade máxima celular de $1050 \mathrm{mg} . \mathrm{L}^{-1}$ obtendo uma produtividade de biomassa de $13,19 \mathrm{mg} \cdot \mathrm{L}^{-1} \cdot \mathrm{h}^{-1}$, com uma velocidade máxima específica de crescimento celular 0,02h-1.

A Figura 1 também mostra a concentração total dos compostos orgânicos voláteis (COVs) formados durante as fases de crescimento celular no decorrer do experimento. A formação dos compostos voláteis foi observada a partir da fase exponencial apresentando a maior concentração durante a fase de declínio, isso pode sugerir que a concentração e disponibilidade de nutrientes do meio externo pode afetar o metabolismo secundário das microalgas (Xu et al., 2017).

Figura 1 - Curva de crescimento celular correlacionado com a concentração dos compostos voláteis ao longo do experimento.

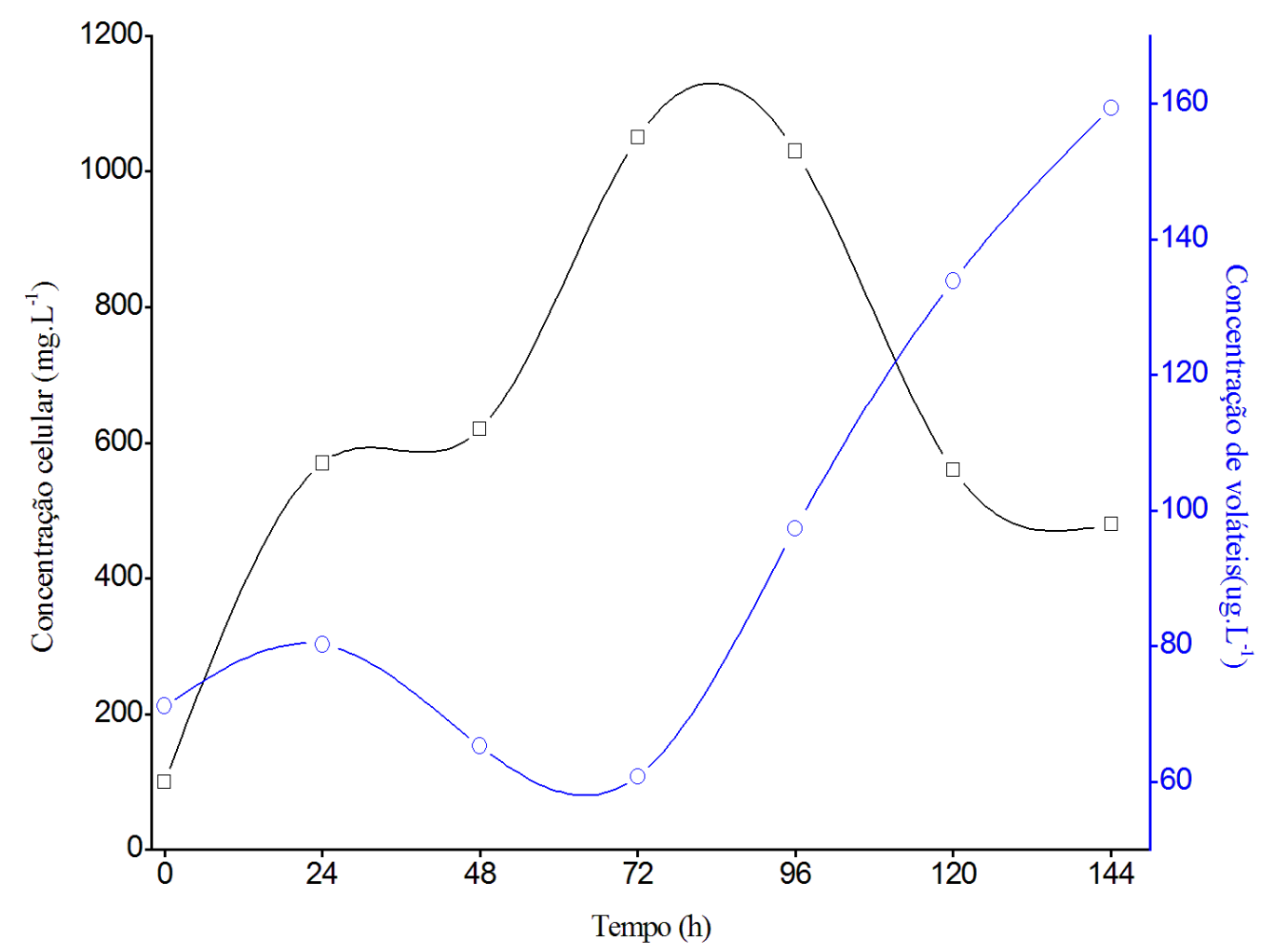

Concentração celular da biomassa microalgas (cor preta); concentração de COVs (cor azul).

A queda na produção dos voláteis observada na transição de fase exponencial para estacionária poderia ser explicada com um descompasso metabólico com o estado nutricional 
(Vidoudez \& Pohnert, 2012; Kolber et al., 1988) e a eficiência na formação voltou após a adaptação do metabolismo celular.

Durante o experimento foi identificado 44 compostos, os que apresentam maior interesse comercial estão listados na Tabela 1, que mostra os valores quantitativos em função da fase de crescimento do microorganismo.

Tabela 1 - Compostos voláteis detectados por GC / MS com seus índices de kovats e o seu interesse industrial.

\begin{tabular}{lcccccc}
\hline Composto & $\begin{array}{c}\text { Indice } \\
\text { de } \\
\text { kovats }\end{array}$ & $\begin{array}{c}\text { Fase } \\
\text { Exponencial } \\
{[\mu / \mathrm{L}]}\end{array}$ & $\begin{array}{c}\text { Fase } \\
\text { Estacionária } \\
{[\mu / \mathrm{L}]}\end{array}$ & $\begin{array}{c}\text { Fase } \\
\text { Declínio } \\
{[\mu / \mathrm{L}]}\end{array}$ & Classe & $\begin{array}{c}\text { Aplicação } \\
\text { industrial }\end{array}$ \\
\hline Limoneno & 1182 & 81,78 & 14,81 & - & Terpeno & Biosolvente \\
2-Nonanone & 1272 & 1,1 & 4,77 & 7,74 & Cetona & Fragrância \\
Mentol & 1642 & 17,20 & 9,97 & 5,12 & Álcool & Aromatizante \\
Benzotiazol & 1896 & 28,78 & 29,18 & 44,38 & Nitrogenado & Farmacêutica \\
Benzofenona & 2437 & 10,05 & 7,21 & 6,63 & Cetona & Fragrância \\
Ftalato de & 2477 & 17,58 & 12,88 & 25,98 & Ester & Plastificante \\
diisobutilo & & & & & & \\
\hline
\end{tabular}

A cinética de formação dos voláteis durante as diferentes etapas de crescimento foi bastante diferenciada entre si. Taxas elevadas de terpenos foram observadas durante o crescimento exponencial e um declínio acentuado na fase de crescimento estacionário, esse fato pode sugerir que houve a interrupção na formação de certos compostos desta classe, isso pode estar envolvido no esgotamento do precursor que não pode ser sintetizado no escuro (Juttner et al., 1983).

\section{CONCLUSÃO}

Os resultados demonstram que as principais fases de crescimento celular para a formação dos compostos orgânicos voláteis em biorreator heterotrófico com água residuária foram à fase exponencial e de declínio.

\section{REFERENCIAS}

HAVEL, J. AND WEUSTER-BOTZ, D. Comparative study of cyanobacteria as biocatalysts for the asymmetric synthesis of chiral building blocks. Eng Life Sci, 6, p.175-179, 2006.

JUTTNER, F. L. AND MOHREN, S.; Environmental Factors Mecting the Formation of Mesityloxide, Dimethylallylic Alcohol and Other Volatile Compounds Excreted by Anabaena cylindric. Journal of General Microbiology, 129, p.407- 412, 1983.

PLAZA, M.; HERRERO, M.; CIFUENTES A. A.; IBÁÑEZ, E. Innovative natural functional ingredients from microalgae. Journal of Agricultural and Food Chemistry, 57 (16), p.7159-7170, 2009. 
POHNERT, G. AND VIDOUDEZ, C.; Comparative metabolomics of the diatom Skeletonema marinoi in different growth phases. Metabolomics, 8, p.654- 669, 2012.

RIPPKA, R.; DERUELES, J.; WATERBURY, J.B.; HERDMAN, M.; STANIER, R.Y. Generic assignments strain histories and properties of pure cultures of cyanobacteria. Journal of General and Microbiology, 111, p.1-61, 1979.

SANTOS, A.B.; FERNANDES, A.F.; WAGNER, R.; JACOB-LOPES, E.; ZEPKA, L.Q. Biogeneration of volatile organic compounds produced by Phormidium autumnale in heterotrophic bioreactor. Journal of Applied Phycology 1, p.1-10, 2016.

SHIPIN, O. AND MEIRING, P. Pond(s) integrated with trickling filter and activated sludge processes. Pond Treatment Technology, London, 2005.

SU, Y.; MENNERICH, A.; URBAN, B. Comparison of nutriente removal capacity and biomass settleability of four high-potential microalgal species. Bioresource Technology, 124, p.157-162, 2012.

XU, Q. ; YANG, L. ; YANG, W. ; BAI, Y. ; HOU, P. ; ZHAO, J. ; ZHOU, L. ; ZUO, Z. Volatile organic compounds released from Microcystis flos-aquae under nitrogen sources and their toxic effects on Chlorella vulgaris. Ecotoxicology and Environmental Safety, 135, p.191-200, 2017. 